\title{
Stimulus probability and sequential effect in recognition memory*
}

\author{
RICHARD S. MARKEN and ARTHUR J. SANDUSKY \\ University of California, Santa Barbara, California 93106
}

\begin{abstract}
The bias for reporting "old" in a recognition-memory task varied inversely with the probability of presenting a previously studied item. The bias for an "old" response is also greater when the stimulus on the preceding trial was a new item. These results invalidate the assumptions of the theory of signal detection for recognition memory and indicate a need for sequential analysis of recognition memory data to obtain unambiguous measures of bias and retention.
\end{abstract}

The theory of signal detection (TSD) has been applied to the study of recognition memory as a method for obtaining unbiased estimates of retention (Banks, 1970). In the typical recognition memory experiment, Os judge whether a previously studied (old) or novel (new) stimulus is presented on each trial. The theory assumes that each stimulus presentation produces a familiarity value, and the $\mathrm{O}$ reports the occurrence of an old stimulus if this value exceeds a fixed-decision criterion. The, value of the criterion determines the Os' bias for reporting the occurrence of old stimuli. The measure of retention is $\mathrm{d}^{\prime}$, the normalized difference between the distributions of familiarity values for old and new stimuli. If the assumptions of the theory are satisfied, $d^{\prime}$ remains invariant with changes in the value of the criterion.

TSD assumes that the criterion is set so as to maximize correct responses and minimize false recognition responses consistent with the a priori stimulus probabilities. The resulting bias is called probability assimilation, because there is a tendency to identify both stimuli with the response appropriate to the more probable type of stimulus.

It is not clear whether this assumption is applicable to recognition memory. Parks (1966) found assimilation in recognition memory experiments when informational feedback was provided about the correct response on each trial. The results of psychophysical studies show that assimilation occurs only for signal detection when Os are explicitly informed of the stimulus probabilities, either by instructions or feedback after each response (Friedman et al, 1968). However, in sensory-recognition tasks for which feedback is not given, response bias is inversely related to stimulus probabilities (Tanner et al, 1970; Sandusky, 1971). This type of bias is called contrast because there is a tendency to identify both stimuli with the response appropriate to the less probable stimulus.

The different bias effects in psychophysical experiments may depend on the ability to learn stimulus

*This research was supported by Public Health Service Grant PHS-MH-21983-01. probabilities. The presence of an immediate standard for comparative judgment appears to be the crucial variable which determines the ability to learn stimulus probabilities (Kinchla, 1966). In detection experiments, continuous background noise serves as the standard, while in recognition experiments, the $\mathrm{O}$ must rely on the memory for prior stimuli as a standard for judgment (Sandusky \& Ahumada, 1971). Insofar as memory for prior stimuli is distorted, it serves as a biased standard.

In recognition memory no obvious standard exists for comparing the familiarity of a stimulus to determine if it is old or new. When the $\mathrm{O}$ is not informed of the stimulus probabilities, the expected bias should be contrast, as in perceptual recognition tasks. The present experiments investigate bias in recognition memory experiments when Os are given no information about stimulus probabilities.

\section{METHOD}

The Os for this experiment were 12 students in introductory psychology at the University of California, Santa Barbara. A continuous recognition memory task (Shepard \& Teghtsoonian, 1961) was performed in which the Os were presented a series of stimuli which included old and new items. The stimuli were three-digit numbers. The stimulus series was constructed so that an old item was presented only once after its initial presentation. From 3 to 9 stimuli intervened between the first and second presentation of an old item. Os went through two separate series of 196 stimuli at their own pace. The Os were instructed to judge whether or not each stimulus had occurred previously in the deck. They were to respond "old" if they remembered seeing the number in the deck and "new" otherwise. The probability of an old stimulus, $\operatorname{Pr}$ (old), was .5 in one series and .2 in the other. Six Os performed in the .5 probability condition and six performed in the .2 condition.

\section{RESULTS AND DISCUSSION}

The mean conditional probabilities of the response "old" to old stimuli (hit) and "old" to new stimuli (false recognition) in the two probability conditions are shown on a receiver operating characteristic (ROC) graph in Fig. 1. The point for the condition in which the probability of an old item was high (.5) is below and to 


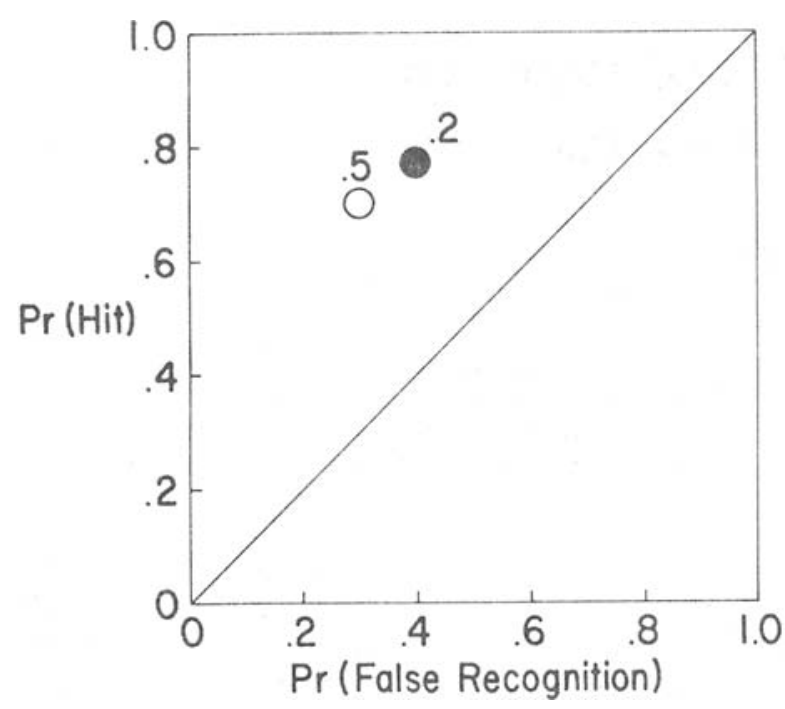

Fig. 1. ROC graph of average conditional-response probabilities for $12 \mathrm{Os}$ in two stimulus probability conditions. The point for the condition in which $\operatorname{Pr}($ old $)=.2$ is above and to the right of the point for $\operatorname{Pr}($ old $)=.5$, indicating a shift in response bias away from the response "old" with greater probability of old stimuli.

the left of the point for the condition in which the probability of an old item was low. This indicates that the bias for responding "old" is inversely related to the probability of an old stimulus. This probability contrast is opposite to the bias effect predicted by TSD if Os know the stimulus probabilities.

The observed contrast could result from a tendency

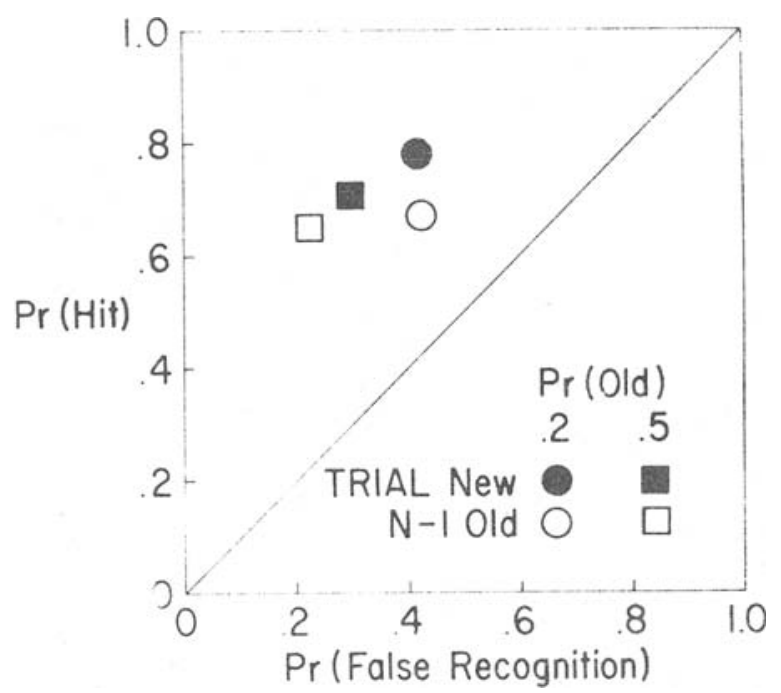

Fig. 2. Sequential ROC graph of average response probabilities for Os in two probability conditions. The points are observed probabilities on Trial $\mathrm{n}$ as a function of the stimulus event (old or new) on Trial n-1. Bias for the response "old" is greater when the prior stimulus is new indicating sequential stimulus contrast. for Os to place the bias so as to use responses with equal frequency (Parducci, 1965), or from response bias determined by the prior sequence of stimuli (Sandusky, 1971). A strong tendency for responses to contrast with prior stimulus values (sequential stimulus contrast) would infirm the assumptions of TSD used to obtain a separate measure of retention. The data were analyzed to determine the sequential dependencies between responses and prior stimuli.

The first order sequential stimulus dependencies are presented in Fig. 2. The ROC shows the conditional probabilities of the response "old" on Trial $n$ as a function of the stimulus value on Trial $n-1$ for the two stimulus probability conditions. For both probability conditions, the point representing the prior occurrence of new stimuli is above and to the left of the point representing prior occurrence of old stimuli, indicating sequential stimulus contrast. The significance of the effect of prior stimuli on bias was determined by computing, for each $\mathrm{O}$, first-order sequential bias scores using the formula:

$$
\begin{aligned}
& \text { "Old" bias } \\
& \quad=\operatorname{Pr}\left(\text { hit } / \mathrm{O}_{n-1}\right)+\operatorname{Pr}\left(\text { false recognition } / \mathrm{O}_{n-1}\right) \\
& \quad-\operatorname{Pr}\left(\text { hit } / \mathrm{N}_{\mathrm{n}-1}\right)-\operatorname{Pr}\left(\text { false recognition } / \mathrm{N}_{\mathrm{n}-1}\right)
\end{aligned}
$$

where $\mathrm{O}_{n-1}$ and $\mathrm{N}_{n-1}$ indicate an "old" and a "new" stimulus on the previous trial, respectively.

Ten of the $12 \mathrm{Os}$, 5 in each probability condition, had negative scores, showing significant first-order sequential contrast. However, both points for the .2 condition are above and to the left of the respective points for the .5 condition, indicating that the probability bias effect is not completely accounted for by the sequential effect.

The present experiments tested whether two important assumptions of TSD are satisfied in recognition memory tasks. One assumption is that Os bias responses in order to optimize correct responding. The other assumption is that the response on each trial is independent of the stimulus on prior trials. TSD predicts that Os will increase their bias for responding that the stimulus presented is "old" as the probability of occurrence of an old stimulus increases. This was not found in the present study.

The results indicate the need for reporting recognition memory results in terms of ROC graphs, such as Fig. 2, which factor out sequential dependencies. Sequential effects can inflate or depress measured retention (Sandusky, 1971), making TSD measures like $\mathrm{d}^{\prime}$ questionable measures of retention.

\section{REFERENCES}

Banks, W. P. Signal detection theory and human memory. Psychological Bulletin, 1970, 74, 81-99.

Friedman, M. P., Carterette, E. C., Nakatani, L., \& Ahumada, A. Comparisons of some learning models for response bias in signal detection. Perception \& Psychophysics, 1968, 3, 5-11. 
Kinchla, R. A. A comparison of sequential effects in detection and recognition, Psychology Department, New York University, Exp. Psychol. Ser. Tech. Rep., 1, 1966.

Parducci, A. Category judgment: A range-frequency model. Psychological Review, 1965, 72, 407-418.

Parks, T. E. Signal detectability theory of recognition memory performance. Psychological Review, 1966, 73, 44-58.

Sandusky, A. Signal recognition models compared for random and Markov presentation sequences. Perception \& Psychophysics, 1971, 10, 339-346.

Sandusky, A., \& Ahumada, A. Contrast in detection with gated noise. Journal of the Acoustical Society of America, 1971, 49, 1790-1794.

Shepard, R. N., \& Teghtsoonian, A. Retention of information under conditions approaching a steady state. Journal of Experimental Psychology, 1961, 62, 302-309.

Tanner, T. A., Rauk, J. A., \& Atkinson, R. C. Signal recognition as influenced by information feedback. Journal of Mathematical Psy chology, 1970, 7, 259-274.

(Received for publication April 18, 1974.)

\title{
Communal housing and shock-induced aggression*
}

\author{
THOMAS L. CREER \\ Children's Asthma Research Institute and Hospital, 3401 West 19th Avenue, Denver, Colorado 80204
}

\begin{abstract}
The effect of communal housing on shock-induced aggression was investigated. Testing, which began after 18 rats had been evenly divided and confined to one of three communal cages for 30 days, indicated that only one pair from each of the three cages exhibited high rates of fighting. The other two pairs in each cage, on the other hand, displayed low and variable rates of aggression. In a second experiment, the animals in each communal cage were paired in round-robin fashion with all cagemates. The results showed that Ss displaying higher rates of aggression in Experiment I again exhibited high frequencies of fighting. Rates displayed by other Ss on the other hand, were a function of the particular pairing. Thus, some rats fought when confronted with a more aggressive protagonist. Others, however, did not fight, regardless of the composition of the match.
\end{abstract}

Recent studies (Hutchison, Ulrich, \& Azrin, 1965; Creer \& Powell, 1971) have demonstrated that the conditions under which rats are housed affect the rate of shock-induced aggression exhibited by these animals. For example, Hutchinson and his colleagues (1965) reported that rats housed in a group showed higher rates of shock-elicited fighting during a single test session than did rats housed individually. However, when tested over a number of sessions, the frequency of such aggression stabilized more rapidly for pairs of Ss housed in separate cages and brought together only in the experimental chamber than for animals both caged and tested together (Creer \& Powell, 1971). Several other investigations have demonstrated that mice housed individually show higher attack frequencies towards conspecifics in a no-shock test situation than do nonisolated mice (e.g., Welch \& Welch, 1969).

Another line of research has shown the existence of two subpopulations of rats with regard to shock-induced aggression (Powell, Francis, Braman, \& Schneiderman, 1969; Creer, 1973). Most paired rats fight at near maximum levels to shock. Other rats, however, show little or no shock-elicited fighting. These individual differences have previously been related to differential training histories (Powell, Francis, Francis, \&

\footnotetext{
* This research was supported in part by Grant No. 19884
} from the National Institute of Mental Health.
Schneiderman, 1972). In a more natural situation, such differences may be related to social conditions existing during crucial periods of development. For example, animals housed together for a period of time may develop dominance hierarchies which later influence rates of shock-induced fighting.

The purpose of the present study was twofold: In Experiment I, rats that had lived together in group cages for 30 days were randomly paired and tested in the shock chamber for several sessions. A second experiment was conducted to determine if the relationships noted in the initial experiment would be observed when Ss were paired in a round-robin manner.

\section{GENERAL METHOD}

\section{Apparatus}

A Grason-Stadler sound attenuated rat chamber, in which the inner enclosure measured $11 \frac{1 / 2}{2}$ by $9 \frac{1 / 4}{4}$ by $7-5 / 8$ in., with two sides constructed of metal and the other two of clear plastic, was used. Shock from a Grason-Stadler No. 1964 GS shock source, equipped with a shock scrambler, was delivered to a grid floor consisting of stainless steel rods, spaced $1 / 2$ in. apart. The door to the insulated test cubicle containing the test chamber was removed to permit an unobstructed view of the animals through the Plexiglas door of the inner chamber. The various stimulus conditions were programmed remotely by a modular programming system. The shock parameters were monitored regularly by attaching a meter to the grid floor and measuring the electrical current. The grid floor was wiped clean after each 